\title{
A Multi-Camera Tracker for Monitoring Pedestrians in Enclosed Environments
}

\author{
Xusheng $\mathrm{Wu}$ \\ Computing and Information Systems \\ The University of Melbourne \\ Parkville, VIC 3010, Australia \\ xushengw1@student.unimelb.edu.au
}

\author{
Stephan Winter \\ Infrastructure Engineering \\ The University of Melbourne \\ Parkville, VIC 3010, Australia \\ winter@unimelb.edu.au
}

\author{
Kourosh Khoshelham \\ Infrastructure Engineering \\ The University of Melbourne \\ Parkville, VIC 3010, Australia \\ k.khoshelham@unimelb.edu.au
}

\begin{abstract}
Multi-camera pedestrians tracking is a challenging computer vision task. We propose a multi-camera tracker for monitoring pedestrians in an enclosed shopping environment. We assess the performance of the multi-camera tracker in a case study, tracking customers in a food and speciality market hall. Our multi-camera tracker tracks customers' walking between the stalls in the market. The information is useful for market management, visitor safety, and other potential application areas.
\end{abstract}

Index Terms-Computer vision, Multi-camera tracking, Shopping, Market, Customer behaviour

\section{INTRODUCTION}

Computer vision based tracking is gaining importance. For example, it can be used for pedestrian counting and density estimation, for flow analysis, and for event recognition. Practical examples have been shown as well: in the form of intelligent surveillance systems to help police (Sultani et al. 2018), tracking athletes for game analysis (Pers and Kovacic. 2000), or no-salesman-payment stores based on tracking and analyzing customers ( $\mathrm{Lu}, 2018)$.

Our research focuses on tracking customers in enclosed shopping environments. We construct a multi-camera tracker for these environments, test the tracker, and study the possible applications of the data derived. To support space management and demonstrate applications of such a tracker we present also (a) a simulation software to visualize the extracted crowd movement, and (b) an application to extract customers' dwelling time at shops, where the dwelling time can be an indicator for the customers' intentions. We will show that pedestrians can be tracked over multiple, overlapping video frames, for their trajectory and activity analysis.

The paper presents a case study on tracking customers in a market hall of Queen Victoria Market (QVM), Melbourne. Customers in the market are walking around to explore or buy some products. For our research we had access to the surveillance videos of three cameras in the market hall, with overlapping scenes. The collected videos cover three hours, starting in the early morning when QVM just opened to the public. The market is not crowded at this time.

The paper is organized as follows. Section 2 summarizes existing studies of underlying components and multi-camera trackers. Section 3 introduces our proposed methodology. Section 4 shows the evaluation results of our constructed multicamera tracker. Section 5 discusses potential applications using our multi-camera tracker. Section 6 summarizes our project contribution.

\section{RELATED WORK}

There is abundant literature on computer vision tracking tasks. Most of it focuses on developing models working on public benchmark datasets. Currently, the most significant benchmark is the MOTChallenge published by Milan et al. (2016). The MOTChallenge dataset covers both dynamic and static camera scenes. It consists of videos captured in different real-world environments. However, the MOTChallenge dataset is a single-camera view dataset, while our research addresses the challenge of tracking in multi-camera monitoring videos. Therefore, existing public single-camera trackers are not enough to solve our research problem.

There is also some research on multi-camera tracking datasets. DukeMTMC, a multi-camera multi-target (MTMC) dataset, is a set of well-annotated, calibrated multi-camera videos recorded on the Duke University campus using eight synchronized cameras. DukeMTMC is recorded in an open non-overlapping environment, while QVM is in an enclosed environment with overlapping video scenes. Accordingly, research on DukeMTMC is also different from ours.

However, the research on such public benchmarks can provide valuable sources for building our multi-camera tracker. Research can be divided into three directions, (a) improving techniques for extracting appearance features, (b) extracting compensation features (such as motion features), and (c) developing better detection association methods.

Most research focuses on improving techniques for extracting pedestrians' appearance features. Previously, handcrafted feature extraction methods like the scale-invariant feature transform (SIFT) and the histogram of oriented gradients (HOG) were widely used. While they are tailored and fast, at present, deep learning methods dominate feature extraction. Deep learning methods firstly learn a feature extraction algorithm from abundant training data and then use the algorithm to extract features. Therefore, deep learning methods require more time and stronger computation resources. However, deep 
learning feature extraction has superior performance compared to the traditional feature extraction methods. At present, almost all new proposed computer vision models use deep learning. Among them, deep convolutional neural networks (CNN) are currently widely used. A popular CNN architecture is ResNet He et al. (2016), which is used as a backbone for feature extraction in various applications.

Bhuiyan et al. (2018) learn feature extraction using cumulative weighted brightness transfer functions (CWBTFs) to model appearance variations. Sun et al. (2018) propose a part based convolution (PBC) network. It averages a feature map obtained through the backbone into several parts. Each part is processed with an average pooling to obtain representative part-level fine-grained features. The network then combines these part-level features as the final features. Wang et al. (2018) focus on informative spatial relations of pedestrians. They utilize a spatial attention layer to re-introducing spatial relations in the final classification layer Wang et al. (2018). Wang et al. (2019a) propose a two-stream spatial-temporal network to extract both visual semantic information and depth information. They developed a logistic smoothing technique to fuse extracted features.

Some research focuses on extracting motion features or pose features as a compensation for appearance features. Ristani and Tomasi (2018) use identities' speed difference and directions as motion cues. Tang et al. (2017) extract human body pose features by an OpenPose network and fused these features with appearance features. Wang et al. (2019b) predict the current position with a Kalman filter and use the position difference between predicted and realistic as a compensation feature.

Detection association is another trend in current research. Detection association is often formulated as a graph processing problem. For example, Yoon et al. (2019) formulate the association problem as a one-to-one assignment problem and solved it by the Hungarian algorithm. Chu and Ling (2019) formulate the problem as a multi-dimensional assignment (MDA) problem, which has a better generalization performance. Ristani and Tomasi (2018) formulate the problem as a graph partition problem, solved by a binary integer program. Jiang et al. (2018) modelled the tracking problem as a person reidentification problem. They extract single-camera view identities' appearance features and calculated the distance between the appearance features of identities in different camera views. A pair of identities with a distance in feature space below a threshold is considered as the same identity. Most research focuses on the detection and tracking of individual pedestrians. However, in crowded scenes, it is difficult to detect and track individuals. Therefore, some approaches measure congestion as a whole. For example, Li et al. (2020) proposed a multiview crowd congestion map fusion framework for macroscopic crowd congestion management. The proposed model addresses the detection difficulties in occluded views, which could also inspire individuals tracking.

\section{Methodology}

The structure of our multi-camera tracker has three components. The tracker firstly extracts single-camera view identities using a public single-camera tracker. Then an appearance feature extractor and motion feature extractor extracts features of these identities. These features are fused together across multiple camera views by constructing an affinity graph. Figure 1 shows the structure of the proposed tracker. The rest of this section introduces the methods in detail.

\section{A. Single Camera Tracker}

We use the single camera tracker proposed by Bergmann et al. (2019). It links detected pedestrians across frames by their bounding box regression algorithm. Compared with other linking methods, this method is comprehensible by human visual inspection. Algorithm 1 introduces the principles of this method in detail.

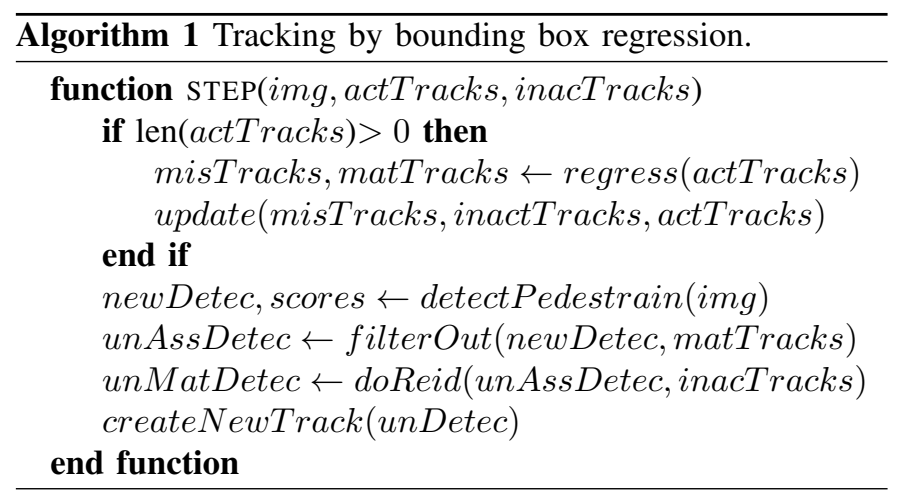

The parameter img represents the current frame. The inactTracks is an array recording those tracks that do not find a match in the previous frame. The actTracks is an array recording tracks that find a match in the previous frame. The regress function regresses the bounding box position of each actTrack firstly. Those highly confident regressed positions are marked as 'matched' while others are marked as 'mismatched'. The update function removes 'mismatched' tracks from actTracks and adds them into inactTracks. The filterOut function is a non-maximum suppression (NMS) function, in essence. The function will remove 'matched' tracks from new detections, and those left are marked as 'unAssigned'. For each 'unAssigned' detection, the algorithm then tries to find a match in the 'mismatched' tracks by a re-identification operation. Those detections that cannot find a match via re-identification will be initialized as new tracks.

This tracker had been proposed for the MOTChallenge dataset. Our QVM dataset is different to the MOTChallenge dataset in that it represents an enclosed (shopping) environment. Therefore, we manually tune the hyperparameters of the single-camera tracker to adapt it to the QVM dataset.

\section{B. Multi-camera Identitie Association Method}

We formulate identity association as a graph assignment problem or graph cut problem. Though the QVM dataset is an overlapping camera scenario, we formulate the problem for both overlapping and non-overlapping scenarios. The 


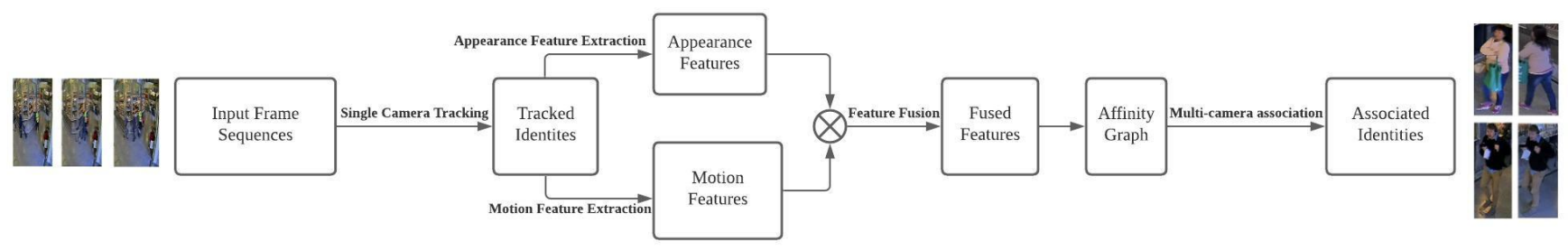

Fig. 1: Proposed model structure.

algorithm firstly constructs the affinity graph using single camera tracked identities. The single camera tracked identities become vertices of the affinity graph, and the affinities between identities become the weights of the graph edges. Affinity can be computed by different methods. Table 1 defines the notations that are used to calculate the affinity.

\begin{tabular}{|l|l|}
\hline Notation & Description \\
\hline$T$ & Set of all camera trajectories \\
\hline$T^{\prime}$ & $\begin{array}{l}\text { Trajectories satisfying motion feature con- } \\
\text { straints; } T^{\prime}=m f(T)\end{array}$ \\
\hline$m f$ & Motion feature constraints \\
\hline$a f$ & Appearance feature extractor \\
\hline dist $_{a p p}\left(t_{1}, t_{2}\right)$ & $\begin{array}{l}\text { Euclidean distance between appearance fea- } \\
\text { tures given any two trajectories }\end{array}$ \\
\hline dist $_{r w}\left(t_{1}, t_{2}\right)$ & Real world distance of two identities \\
\hline$a y\left(t_{1}, t_{2}\right)$ & Affinity value of any two given trajectories \\
\hline
\end{tabular}

TABLE I: Notations in affinity definitions.

1) Feature Extraction: We use a pre-trained Siamese network to extract identity appearance features, and develop a motion model for the multi-camera tracker. The motion features used are related to the camera layout. There are three cameras in our dataset, as shown in Figure 2 They are labelled 136, 137, and 139 respectively. Cameras 136 and 137 overlap, and cameras 139 and 137 overlap. There is a $20 \mathrm{~ms}$ time difference between camera 136 and camera 137, and $20 \mathrm{~ms}$ time difference between camera 137 and camera 139; however, we have synchronized them. Pedestrians can walk into the range of cameras from four directions, as indicated by arrows.

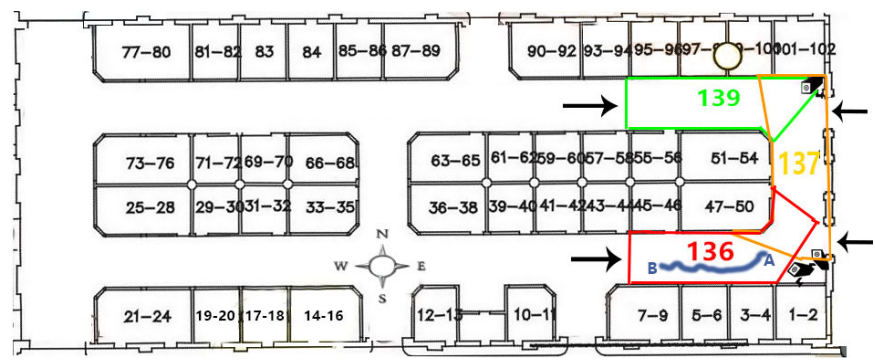

Fig. 2: Layout of the cameras in the QVM dataset.

The first motion cue we used is the straight line walking direction of customer, which we have simplified in this grid environment to North, South, East, or West. The direction is represented as a vector: $(0,1)$ represents North, $(1,0)$ represents East, $(0,-1)$ represents South, and $(-1,0)$ represents West. We assume that pedestrians would not walk in circles, that means pedestrians would not walk directly from camera 136 to 139 without passing camera 137 , and versa vice. The blue curve in Figure 2 is an example of a pedestrian trajectory. The pedestrian walked from $\mathrm{A}$ to $\mathrm{B}$. The straight-line direction is West $(-1,0)$.

The straight-line walking direction motion cue can be described in detail as follows:

a) Pedestrian with direction $(-1,0)$ in camera 136 can only match pedestrian with direction $(0,-1)$ in camera 137.

b) Pedestrian with direction $(1,0)$ in camera 136 can only match pedestrian with direction $(0,1)$ in camera 137 .

c) Pedestrian with direction $(1,0)$ in camera 139 can only match pedestrian with direction $(0,-1)$ in camera 137 .

d) Pedestrian with direction $(-1,0)$ in camera 139 can only match pedestrian with direction $(0,1)$ in camera 137 .

Besides the straight-line direction, the motion model also uses the spatial-temporal and speed constraints. In the case of non-overlapping cameras,spatial-temporal cues and speed constraints are:

a) Two trajectories that occur in different cameras at the same time are marked as impossible matching.

b) Two identities with speed differences higher than 30 units are marked as an impossible matching. 30 units is an estimated statistical value based on observations.

In the overlapping case, spatial-temporal cues and speed constraints are:

a) A trajectory not passing through the overlapping area should not have any matching.

b) Two trajectories not overlapping on the timeline are an impossible matching.

The motion feature filter will remove those impossible matchings. Those left candidates will construct an affinity graph by fusing the distances in appearance features and in real-world position.

2) Graph Assignment Problem: We formulate the identity association as a graph assignment problem: Given two single camera tracking identities set, $T 1$ and $T 2$, together with an affinity function ay: $T 1 \times T 2 \rightarrow R$, find a bijjection $f$ : $T 1 \rightarrow T 2$ such that the objective function

$$
\sum_{t 1 \in T 1, f(t 1) \in T 2} a y(t 1, f(t 1))
$$

is minimized. 
In the non-overlapping case, the affinity function is defined as:

$$
\begin{gathered}
\text { ay }\left(t_{1}, t_{2}\right)= \begin{cases}\text { dist }_{\text {app }}\left(t_{1}, t_{2}\right) & \forall t_{1}, t_{2} \in T^{\prime} \\
\infty & \text { otherwise }\end{cases} \\
\text { where } t_{2}=f\left(t_{1}\right)
\end{gathered}
$$

In the overlapping case, the affinity function is defined as:

$$
\begin{aligned}
\operatorname{ay}\left(t_{1}, t_{2}\right) & = \begin{cases}\frac{\text { dist }_{a p p}\left(t_{1}, t_{2}\right) \cdot \text { dist }_{r w}\left(t_{1}, t_{2}\right)}{7 \operatorname{dist}_{a p p}\left(t_{1}, t_{2}\right)+10 \text { dist }_{r w}\left(t_{1}, t_{2}\right)} & \forall t_{1}, t_{2} \in T^{\prime} \\
\infty & \text { otherwise }\end{cases} \\
\text { where } & t_{2}=f\left(t_{1}\right)
\end{aligned}
$$

We solve the graph assignment problem by invoking the solver provided in this website

3) Graph Cut Problem: We formulate the identity association as graph cut problem: Given the two sets of single camera tracking identities $T_{1}, T_{2}$, these identities are connected with each other by affinity, which is a similarity measure between identities. The connection is represented in a graph $G(V, E)$, with $a y: E \rightarrow R$. The graph cut problem will cut some edges to maximize affinity of $G$. The graph cut can be mathematically described as:

$$
\begin{aligned}
\text { maximize } & \sum_{e \in E} c_{e} \cdot a y(e) \\
\text { where } & c_{e} \in\{0,1\}
\end{aligned}
$$

$c_{e}=0$ means cutting the edge, while $c_{e}=1$ means keeping the edge.

In the non-overlapping scenario, the affinity function is defined as:

$$
a y\left(t_{1}, t_{2}\right)= \begin{cases}\frac{1}{\operatorname{dist}_{a p p}\left(t_{1}, t_{2}\right)} & \forall t_{1}, t_{2} \in T^{\prime} \\ -\infty & \text { otherwise }\end{cases}
$$

In the overlapping scenario, the affinity function is defined as:

$$
a y\left(t_{1}, t_{2}\right)= \begin{cases}\frac{10}{\operatorname{dist}_{a p p}\left(t_{1}, t_{2}\right)}+\frac{\text { dist }_{r w}\left(t_{1}, t_{2}\right)}{7} & \forall t_{1}, t_{2} \in T^{\prime} \\ -\infty & \text { otherwise }\end{cases}
$$

We solve the graph cut problem by invoking the solver provided in this website

\section{EXPERIMENTAL EVALUATION AND RESUlts}

\section{A. Evaluation Metrics}

We use three evaluation metrics. This includes multi-object tracking accuracy (MOTA), multi-object tracking precision (MOTP) and identity F1 (IDF1). The higher the value of these metrics, the better the performance. Their respective calculation formulas are as follows:

$$
\begin{aligned}
M O T A & =1-\frac{\sum_{t}\left(F N_{t}+F P_{t}+I D S W_{t}\right)}{\sum_{t} G T_{t}} \\
M O T P & =\frac{\sum_{t, i} d_{t, i}}{\sum_{t} c_{t}} \\
I D F 1 & =\frac{2 I D T P}{2 I D T P+I D F P+I D F N}
\end{aligned}
$$

These three metrics are a subset of the MOTChallenge benchmark evaluation tool. The MOTA metric combines the number of false positives (FP), false negatives (FN), and identity switches (IDSW). The MOTP metric reflects the misalignment between annotations and predictions. The parameter $c_{t}$ represents the number of matches between annotations and detections in the $t$ frame. The parameter $d_{t, i}$ is the position distance of the $i$ th's pair in frame $t$ The IDF1 metric combines the number of identity true positives (IDTP), identity false positives (IDFP), and identity false negatives (IDFN).

\section{B. Single Camera Tracking Evaluation}

We firstly compare the chosen public single-camera tracker with other state-of-the-art trackers on the public benchmark MOTChallenge.

\begin{tabular}{|c|c|c|c|c|}
\hline \multicolumn{2}{|c|}{ Tracker } & IDF1 & MOTA & MOTP \\
\hline LSST17 & Feng et al. & $62.3 \%$ & $54.7 \%$ & $75.9 \%$ \\
\hline MPNTrack(Braso and Leal-Taixén & $2020)$ & $61.7 \%$ & $58.8 \%$ & $78.6 \%$ \\
\hline HDTR(Babaee et al. & 2018 \\
\hline ChosenTracker(Bergmann et al. & $48.4 \%$ & $54.1 \%$ & $80.2 \%$ \\
\hline
\end{tabular}

TABLE II: Single camera MOTChallenge tracking result comparison.

As shown in Table II] the chosen tracker has a competitive performance compared to other trackers. We further evaluate the chosen tracker on the QVM dataset.

\begin{tabular}{|c|c|c|c|}
\hline Dataset & IDF1 & MOTA & MOTP \\
\hline cam136 & $83.5 \%$ & $76.5 \%$ & $76.8 \%$ \\
\hline cam137 & $66.6 \%$ & $57.8 \%$ & $77.8 \%$ \\
\hline cam139 & $60.5 \%$ & $48.4 \%$ & $76.4 \%$ \\
\hline OVERALLL & $68.6 \%$ & $58.0 \%$ & $77.0 \%$ \\
\hline
\end{tabular}

TABLE III: Single camera MOT tracking result on the QVM dataset.

Table III shows that the chosen public single-camera tracker has better performance on the QVM dataset than on the MOTChallenge dataset. This is because we manually tune the hyperparameters of the chosen public single-camera tracker. The chosen single-camera tracker was initially proposed for MOTChallenge dataset. The MOTChallenge dataset recorded open and dynamic-view scenes, while our QVM dataset collected data in enclosed and static-view scenes. Therefore, we tuned some hyperparameters including the alive time of a disappeared pedestrian and the number of person re-identification features. Besides, different from the original, we also assign a decaying weight for person re-identification features, which means our tracker would focus on the more recent appearance features. The resolution and fps of MOTChallenge dataset and QVM dataset is another obvious difference. For this difference, we tuned the hyperparameter values of person detection threshold and the person re-identification threshold. We tuned hyperparameters by applying a grid search strategy. For each hyperparameter, we used the original model value as the centre and search in its neighbourhood.

\section{Multi Camera Tracking Evaluation}

We implement four different trackers respective to the four different methods discussed above. The trackers are labelled 
in Table IV] Table $\mathrm{V}$ shows their performance comparison. Among these, Tracker 2 has the best performance. Figure 3 and Figure 4 show two correct matching examples by Tracker 2.

\begin{tabular}{|l|l|}
\hline TrackerID & Tracker Description \\
\hline 1 & $\begin{array}{l}\text { Graph Cut Modelling Under Non- } \\
\text { overlapping Scenario }\end{array}$ \\
\hline 2 & $\begin{array}{l}\text { Graph Cut Modelling Under Over- } \\
\text { lapping Scenario }\end{array}$ \\
\hline 3 & $\begin{array}{l}\text { Graph Assignment Modelling Un- } \\
\text { der Non-overlapping Scenario }\end{array}$ \\
\hline 4 & $\begin{array}{l}\text { Graph Assignment Modelling Un- } \\
\text { der Overlapping Scenario }\end{array}$ \\
\hline
\end{tabular}

TABLE IV: Tracker modelling method.

\begin{tabular}{|c|c|c|c|}
\hline TrackerID & IDF1 & MOTA & MOTP \\
\hline 1 & $50.2 \%$ & $46.8 \%$ & $75.9 \%$ \\
\hline 2 & $57.9 \%$ & $57.9 \%$ & $76.2 \%$ \\
\hline 3 & $51.9 \%$ & $57.9 \%$ & $76.2 \%$ \\
\hline 4 & $51.7 \%$ & $57.9 \%$ & $76.2 \%$ \\
\hline
\end{tabular}

TABLE V: Multi-camera MOT tracking result comparison.
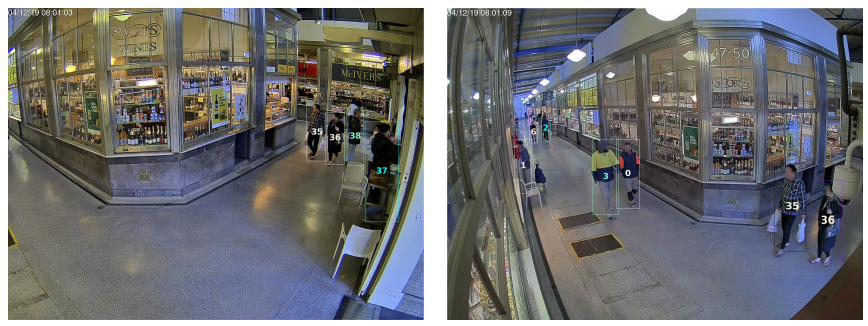

Fig. 3: An example of successful matching: Pedestrians 35 and 36 are detected in both cameras 136 and 137, and the multi-camera tracker correctly matches the detections across the two views.
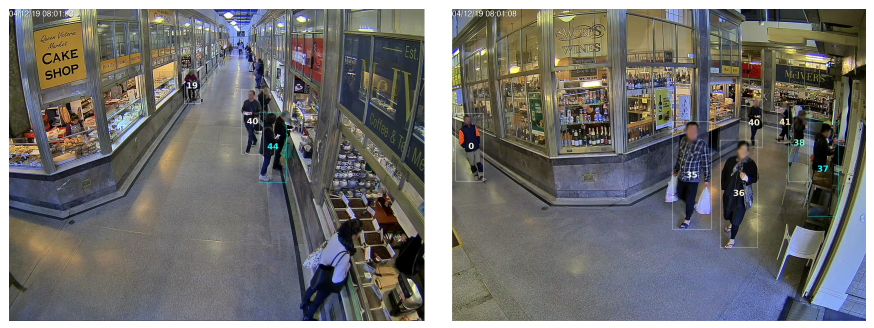

Fig. 4: An example of successful matching: Pedestrian 40 is detected in both cameras 137 and 139, and the multi-camera tracker correctly matches the detections across the two views.

\section{Applications of the Multi-CAMERA Tracker}

We also demonstrate the potential application of our proposed multi-camera tracker. For this purpose, we develop two applications, one application used to infer customers' intentions via trajectory analysis, and one application to simulate customers' movements in a bird's eye visualization.
Pedestrian trajectories reflect their intentions. Customers in QVM are walking along aisles and between stalls to explore or buy some products. Therefore, it is possible to infer what these customers want to buy by analyzing their trajectories. A simple application making this inference uses two cues, the dwelling time of customers at stalls, and and the stalls' business scope. Setting a dwelling time threshold, e.g., 15 seconds, it is reasonable to say that a customer stopping at a stall for a longer period is trying to buy or look for something from that stall. A second threshold is required to determine when a customer stops at a stall - based on the distance of their trajectory from the stall's front window, which, for our case, are visualized in Figure 5 by the red lines.

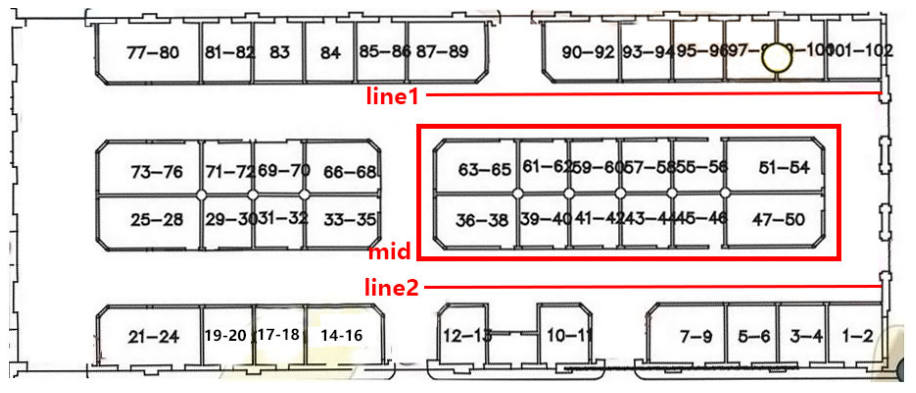

Fig. 5: Distance thresholds for customers dwelling at stalls.

The store business scope is recorded in a database. Figure 6 is an example of our application inference. The system detects Customer 1 stopping at Stall 5-6 for over 15 seconds. The system checks the database and knows that Stall 5-6 mainly sells all kinds of sausages. Therefore, the system infers that Customer 1 is interested in buying some sausage.
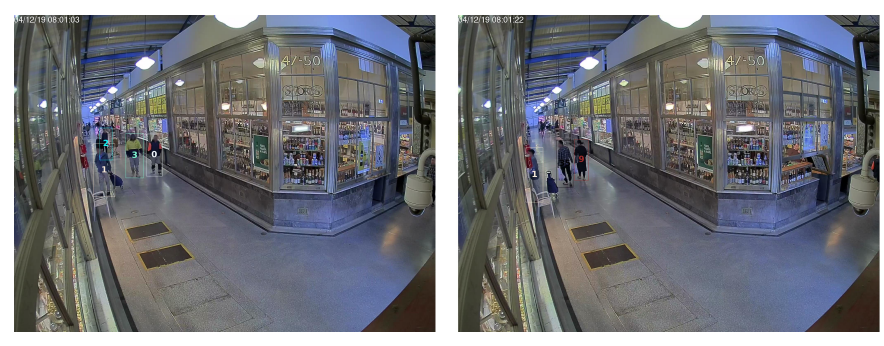

Fig. 6: Our tracker detects Pedestrian 1 stopping by a stall for a long time. The tracker infers that Pedestrian 1 intends to buy something from this stall.

We also develop a visualization tool for customers' movement from a top view. This visualization is more intuitive for human visual analysis of the flow of customers, e.g., for market management, compared to the video streams themselves. Figure 7 shows snapshots of the developed visualization tool.

\section{CONCLUSION}

In conclusion, we proposed a multi-camera tracker for monitoring pedestrians in an enclosed environment. We validated the multi-camera tracker performance by a case study, 


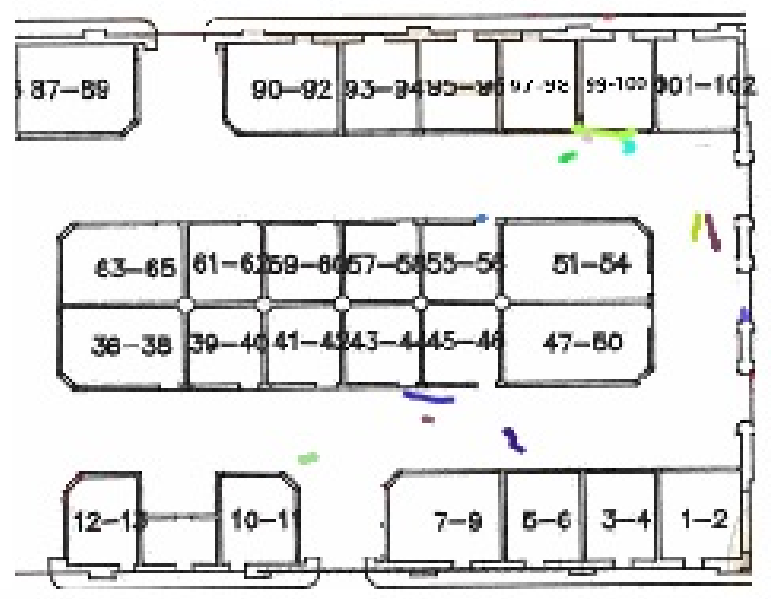

1st snapshot

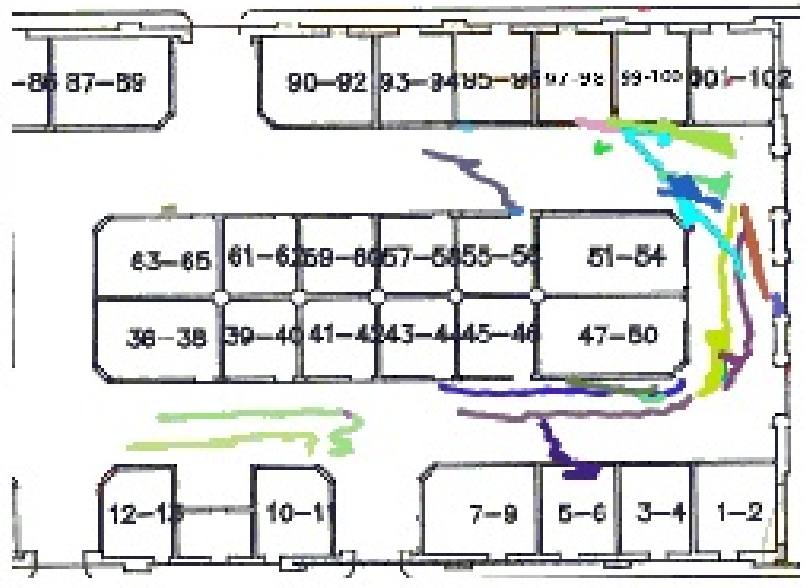

3rd snapshot

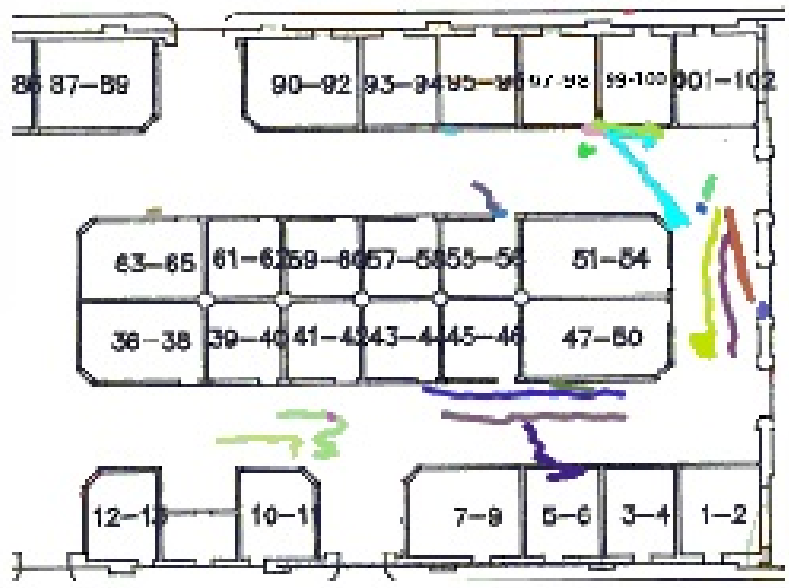

2nd snapshot

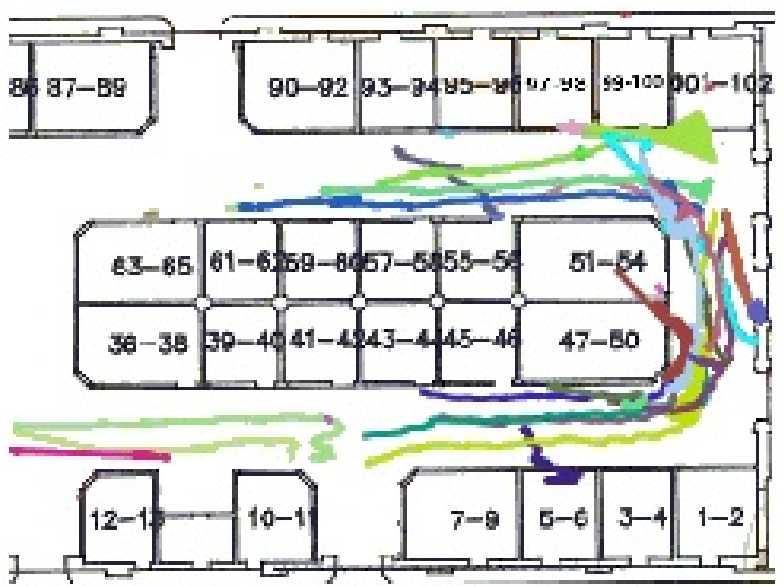

4th snapshot

Fig. 7: Snapshots of customers' movement from bird's-eye perspective. Each colour line represents a pedestrian. The elapsed time between the four snapshots is 8 seconds.

tracking customers in a market hall of QVM. The evaluation results show that our tracker has a competitive performance. The further applications demonstrate that the multi-camera tracker can lay the grounds to develop further tools for space management or customer safety.

There is still work to be done to further improve performance. Our experiments show that the performance of the multi-camera tracker is greatly affected by the single-camera tracker. Therefore, we will focus on improving the singlecamera tracker. In addition, we can explore other multi-camera association methods to make the multi-camera evaluation results more convincing. For example, the correlation clustering method for multi-camera tracking proposed by Ristani and Tomasi (2018) could be explored, which achieved state-ofthe-art performance. In addition, we inferred the customer's intentions by analyzing the tracking results. The inference results need further verification.

\section{ACKNOWLEDGMENT}

We acknowledge the support by QVM by providing access to surveillance video data for this research. The data has been accessed solely for the purpose of this research and cannot be shared. We also acknowledge the use of the deep learning platform provided by the University of Melbourne's High Performance Computing team. 


\section{REFERENCES}

W. Sultani, C. Chen, and M. Shah, "Real-world anomaly detection in surveillance videos," in Proceedings of the IEEE Conference on Computer Vision and Pattern Recognition, 2018, pp. 6479-6488.

J. Pers and S. Kovacic, "Computer vision system for tracking players in sports games," in Proceedings of the First International Workshop on Image and Signal Processing and Analysis. in conjunction with 22nd International Conference on Information Technology Interfaces. IEEE, 2000, pp. 177-182.

L. Lu, "Decoding alipay: mobile payments, a cashless society and regulatory challenges," Butterworths Journal of International Banking and Financial Law, pp. 40-43, 2018.

A. Milan, L. Leal-Taixé, I. Reid, S. Roth, and K. Schindler, "Mot16: A benchmark for multi-object tracking," arXiv:1603.00831, 2016.

K. He, X. Zhang, S. Ren, and J. Sun, "Deep residual learning for image recognition," in Proceedings of the IEEE Conference on Computer Vision and Pattern Recognition, 2016, pp. 770-778.

A. Bhuiyan, A. Perina, and V. Murino, "Exploiting multiple detections for person re-identification," Journal of Imaging, vol. 4, no. 2, p. 28, 2018.

Y. Sun, L. Zheng, Y. Yang, Q. Tian, and S. Wang, "Beyond part models: Person retrieval with refined part pooling (and a strong convolutional baseline)," in Proceedings of the European Conference on Computer Vision (ECCV), 2018, pp. 480-496.

H. Wang, Y. Fan, Z. Wang, L. Jiao, and B. Schiele, "Parameterfree spatial attention network for person re-identification," arxiv:1811.12150, 2018.

G. Wang, J. Lai, P. Huang, and X. Xie, "Spatial-temporal person re-identification," in Proceedings of the AAAI Conference on Artificial Intelligence, vol. 33, 2019, pp. 89338940.

E. Ristani and C. Tomasi, "Features for multi-target multicamera tracking and re-identification," in Proceedings of the IEEE Conference on Computer Vision and Pattern Recognition, 2018, pp. 6036-6046.

S. Tang, M. Andriluka, B. Andres, and B. Schiele, "Multiple people tracking by lifted multicut and person reidentification," in Proceedings of the IEEE Conference on Computer Vision and Pattern Recognition, 2017, pp. 35393548.

Z. Wang, L. Zheng, Y. Liu, and S. Wang, "Towards real-time multi-object tracking,” arXiv:1909.12605, 2019.

Y.-C. Yoon, D. Y. Kim, K. Yoon, Y.-m. Song, and M. Jeon, "Online multiple pedestrian tracking using deep temporal appearance matching association," arXiv:1907.00831, 2019.

P. Chu and H. Ling, "Famnet: Joint learning of feature, affinity and multi-dimensional assignment for online multiple object tracking," in Proceedings of the IEEE International Conference on Computer Vision, 2019, pp. 6172-6181.

N. Jiang, S. Bai, Y. Xu, C. Xing, Z. Zhou, and W. Wu, "Online inter-camera trajectory association exploiting person reidentification and camera topology," in Proceedings of the 26th ACM International Conference on Multimedia, 2018, pp. 1457-1465.

Y. Li, M. Sarvi, K. Khoshelham, and M. Haghani, "Multi-view crowd congestion monitoring system based on an ensemble of convolutional neural network classifiers," Journal of Intelligent Transportation Systems, pp. 1-12, 2020.

P. Bergmann, T. Meinhardt, and L. Leal-Taixe, "Tracking without bells and whistles," in Proceedings of the IEEE International Conference on Computer Vision, 2019, pp. 941-951.

W. Feng, Z. Hu, W. Wu, J. Yan, and W. Ouyang, "Multi-object tracking with multiple cues and switcher-aware classification," arXiv:1901.06129, 2019.

G. Brasó and L. Leal-Taixé, "Learning a neural solver for multiple object tracking," in Proceedings of the IEEE/CVF Conference on Computer Vision and Pattern Recognition, 2020, pp. 6247-6257.

M. Babaee, A. Athar, and G. Rigoll, "Multiple people tracking using hierarchical deep tracklet re-identification," ArXiv, vol. abs/1811.04091, 2018. 


\section{University Library}

\section{- M M N E R VA A gateway to Melbourne's research publications}

Minerva Access is the Institutional Repository of The University of Melbourne

Author/s:

Wu, X;Winter, S;Khoshelham, K

Title:

A Multi-Camera Tracker for Monitoring Pedestrians in Enclosed Environments

Date:

2020-01-01

Citation:

Wu, X., Winter, S. \& Khoshelham, K. (2020). A Multi-Camera Tracker for Monitoring

Pedestrians in Enclosed Environments. Alamaniotis, M (Ed.) Pan, S (Ed.) 2020 IEEE 32ND INTERNATIONAL CONFERENCE ON TOOLS WITH ARTIFICIAL INTELLIGENCE (ICTAI), 2020-November, pp.852-858. IEEE. https://doi.org/10.1109/ICTAI50040.2020.00134.

Persistent Link:

http://hdl.handle.net/11343/268189 